\title{
A study on few biochemical parameters of clinically suspected and laboratory confirmed Leptospirosis cases
}

\author{
D. B. Zala ${ }^{1}$, Vikram Khan ${ }^{2}$, and V.K. Das* \\ *Department of Medicine, Sri Vinoba Bhave Civil Hospital, Dadra \& Nagar Haveli, Silvassa -396230, INDIA \\ ${ }^{1}$ Department of Microbiology, DPHL, VBCH, Directorate of Medical \& Health Services, Dadra \& Nagar Haveli, \\ Silvassa - 396230, INDIA \\ ${ }^{2}$ Directorate of Medical \& Health Services, Dadra \& Nagar Haveli, Silvassa, 396230, INDIA \\ *Corresponding author. E-mail: vkdas511@gmail.com
}

Received: June 30,2012; Revised received: January 7, 2014; Accepted: January 30, 2014

\begin{abstract}
A study on biochemical parameters in clinically suspected and laboratory- confirmed leptospirosis cases was conducted in the Union Territory of Dadra and Nagar Haveli. Out of 50 clinically suspected samples of leptospirosis, 38\% were positive from both ELISA IgM and rapid card test. In the present study, liver markers such as bilirubin, Serum Glutamate Oxalo acetic Transaminase (SGOT) and Serum Glutamate Pyruvic Transaminase (SGPT) encountered elevation $68 \%, 100 \%$ and $89 \%$ respectively in leptospiosis positive patent while $89 \%$ and $48 \%$ of positive patients showed elevation in kidney marker, creatinine and blood urea. On the basis of liver and renal functions, a hospital can develop its own clinical algorithm to suspect the case of leptospirosis.
\end{abstract}

Keywords: Biochemical parameters, Immunochromatogaphy, SGOT, SGPT, Leptospirosis

\section{INTRODUCTION}

Leptospirosis is an infectious disease caused by pathogenic organisms belonging to the genus Leptospira that are transmitted directly or indirectly from animals to humans. Human-to-human transmission of leptospirosis occurs very rarely (Levett, 2001). The infection is commonly transmitted to humans by water contaminated by animal urine to come in contact with unhealed breaks in the skin, the eyes, or with the mucus membrane. Leptospira can cause wide range of clinical manifestations, from a mild, flue-like illness to a severe disease form, characterized by multi-organ system complications leading to death (WHO, 1999). Apart from humans, at least 160 mammalian species are infected like rats, cattle, pigs, buffaloes, horses, sheep, goats, squirrels, bandicoots and raccoons. It is most commonly found in tropical or subtropical countries and may be prevalent in both urban and rural regions. Most outbreaks of leptospirosis are reported in coastal regions: Gujarat, Mumbai, Kerala, Chennai and the Andaman Islands (Meenakshi et al., 2009 and Sethi et al., 2010). It is known that leptospirosis is widespread in farm and domestic animals in many parts of India (WHO, 1999), including the North-East, West Bengal, Bihar, Madhya Pradesh, Maharashtra, Andhra Pradesh, Karnataka, Tamil Nadu, Punjab and Haryana (Charoonruangrit and Boonpucknavig, 1964;
Mamuthausethupathi et al., 1995; Patel et al., 2006; Sugunan et al., 2009 and Velineni et al., 2007). Every year in the season of monsoon, Surat, Navsari, Valsad and Dadra and Nagar Haveli regions of western India are mainly affected from the leptospirosis. The purpose of present investigation, to determine the involvement of liver and kidney in lepspirosis positive patient.

\section{MATERIALS AND METHODS}

Fifty Single or paired samples of clinically suspected leptospirosis patients were collected at Sri Vinoba Bhave Civil Hospital Silvassa during November 2011 to Janury 2012 and tested by Rapid card test working on the principle of immunochromatography, a unique two-site immunoassay on a membrane ( Leptocheck Zypher Inc.). As the test sample flows through the membrane assembly of the test device, the anti-human IgM colloidal gold conjugate forms a complex with IgM antibodies in the sample. This complex moves further on the membrane to the test window ' $\mathrm{T}$ ' where it is immobilized by the broadly reactive Leptospira genus specific antigen coated on the membrane, leading to the formation of a red to deep purple colored band at the test region. ' $\mathrm{T}$ ' which confirms a positive test result. All rapid card screened samples were tested by IgM ELISA for confirmation. All ELISA IgM positive samples were considered as leptospirosis confirmed cases. All biochemical parameters of liver 
D. B. Zala et al. / J. Appl. \& Nat. Sci. 6 (1): 12-13 (2014)

Table 1. Showing abnormality in the function of liver and kidney in Leptospirosis positive patients.

\begin{tabular}{llc}
\hline Function & Parameters & Elevated value in Leptospirosis positive patient (\%) \\
\hline \multirow{2}{*}{ Liver } & SGOT (U/L) & 100 \\
& SGPT (U/L) & 89 \\
\multirow{2}{*}{ Kidney } & Bilirubin (mg/dL) & 68 \\
& Creatinine (mg/dL) & 89 \\
\hline
\end{tabular}

and kidney was tested by fully automated biochemistry analyzer (Xpand Plus, Semens inc)

\section{RESULTS AND DISCUSSION}

Only thirty eight percent of the total clinical suspected cases were found positive for leptospirosis $(n=50)$. The maximum patient of leptospirosis was reported hepatic and renal dysfunction. The elevated indicator of abnormal liver function SGOT, SGPT and bilirubin ware encountered $100 \% 89 \%$ and $68 \%$ in leptospirosis positive patient. In case of abnormality in the function of kidney, it was observed that $89 \%$ patients have elevated creatinine values and $49 \%$ patients have significantly increased values of blood urea (Table 1). It has also been observed that there were abnormal biochemical changes in both the liver as well as renal functions in $89 \%$ of laboratory confirmed cases. The value of abnormality in liver and renal function of laboratory negative cases was lesser in contrast of positive cases.

Leptospirosis is a major public health problem in tropical countries with potentially fatal systemic complications and multiorgan dysfunction, including hepatic and renal failure, with or without severe pulmonary hemorrhage syndrome (Levett et al., 2001). The abnormality due to leptospirosis in the liver and kidney have been reported time to time, In present investigation, the level of abnormality in liver and renal function in leptospirosis confirm cases corresponds with the studies conducted by Sethi et al. (2010), where $73 \%$ patients (63 out of 86 ) were with abnormality in the liver and kidney function. On other hand, only $35 \%$ patients were found with symptoms of abnormality in the liver and kidney by Prabhu et al. (2010), while only three patients (13\%) had abnormality in liver and kidney function during an outbreak in Germany (Desai et al., 2009).

Thus, it was concluded that the abnormal liver and renal function may be considered as an indicator of suspicious case of leptospirosis in highly endemic region and on the basis of elevation of abnormality in the liver and renal functions of suspected case, a hospital can develop their own clinical algorithm towards the confirmation of leptospirosis.

\section{ACKNOWLEDGEMENT}

We thank to the Mission Director (NRHM) UT of Dadra and Nagar Haveli Silvassa, and Integrated Disease Surveillance Programme for provide necessary support.

\section{REFERENCES}

Charoonruangrit, S. and Boonpucknavig, S. (1964). Leptospirosis at Chulalongkorn Hospital: A report of 54 cases. J. of Medical Association. Thailand, 47: 653-659.

Desai, S., van Treeck, U., Lierz, M., Espelage, W., Zota, L. and Sarbu, A. (2009). Resurgence of field fever in a temperate country: an epidemic of leptospirosis among seasonal strawberry harvesters in Germany in 2007. Clinical Infectious Disease, 48: 691-697.

Levett, P. N. (2001). Leptospirosis. Clinical Microbioliology Reviews, 14: 296-326.

Patel, B. K., Gandhi, S. J. and Desai, D. C. (2006). Clinicoepidemiological aspect of leptospirosis in South Gujarat. Indian J. of medical Microbiology, 24(4):322-325.

Mamuthausethupathi, Shivakumar, S., Suguna, R., Jaykumar, M. R., Vijayakumar R, Cor, E. and Carrington, D. G. (1995). Leptospiorosis in Madras - A clinical \& seriological study (1990 - 91) Journal of the Association of Physician India, 43(7):456-458.

Meenakshi, M., Anuradha, De. and Dilip, T. (2009). Leptospirosis outbreak in 2005: L.T.M.G. hospital experience. Indian Journal of Medical Microbiology, 27 (2):153-155.

Prabhu, N., Joseph, P.I.D. and Chinnaswamy, P. (2010). Seroepidemiological trends of human leptospirosis in Coimbatore, India between 2007 and 2009. Advances in Applied Science Research, 1: 113-119.

Sethi, S., Sharma, N., Kakkar, N., Taneja, J., Chatterjee, S.S. and Banga, S.S. (2010). Increasing trends of leptospirosis in northern India: a clinicoepidemiological study. PLOS Neglected Tropical Diseases, 4: 579.

Sugunan, A. P., Vijayachari, P., Sharma, S., Roy, S., Manickam, P., Natarajaseenivasan, K., Gupte, M. D. and Sehgal, S. C. (2009). Risk factors associated with leptospirosis during an outbreak in Middle Andaman, India. Indian Journal of Medical Research, 130(1): 67-73.

Velineni, S., Asuthkar, S., Umabala, P., Lakshmi, V. and Sritharan, M. (2007). Serological evaluation of leptospirosis in Hyderabad, Andhra Pradesh: A retrospective hospital-based study. Indian Journal of Medical Microbiology, 25: 24-7.

WHO (1999). Leptospirosis worldwide, World Health Organization; Wkly. Epidemiol. Rec., 74:237-242. 